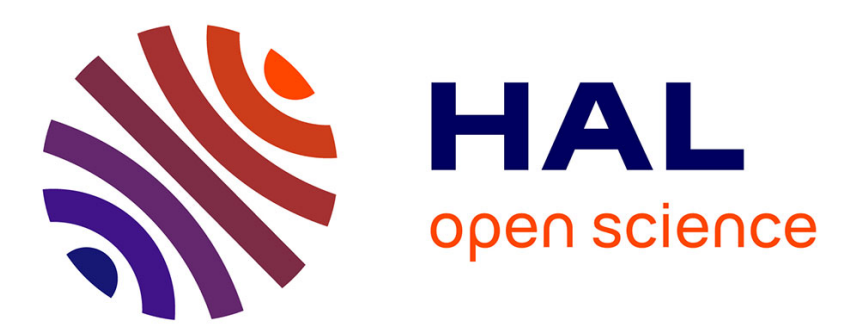

\title{
Solvatochromic Near-Infrared Probe for Polarity Mapping of Biomembranes and Lipid Droplets in Cells under Stress
}

Anila Ashoka, Ashokkumar Pichandi, Yuriy P. Kovtun, Andrey Klymchenko

\section{- To cite this version:}

Anila Ashoka, Ashokkumar Pichandi, Yuriy P. Kovtun, Andrey Klymchenko. Solvatochromic NearInfrared Probe for Polarity Mapping of Biomembranes and Lipid Droplets in Cells under Stress. Journal of Physical Chemistry Letters, 2019, 10 (10), pp.2414-2421. 10.1021/acs.jpclett.9b00668 . hal-02271510

\section{HAL Id: hal-02271510 https://hal.science/hal-02271510}

Submitted on 26 Aug 2019

HAL is a multi-disciplinary open access archive for the deposit and dissemination of scientific research documents, whether they are published or not. The documents may come from teaching and research institutions in France or abroad, or from public or private research centers.
L'archive ouverte pluridisciplinaire HAL, est destinée au dépôt et à la diffusion de documents scientifiques de niveau recherche, publiés ou non, émanant des établissements d'enseignement et de recherche français ou étrangers, des laboratoires publics ou privés. 


\section{Solvatochromic Near-Infrared Probe for Polarity Mapping of Biomembranes and Lipid Droplets in Cells under Stress}

Anila Hoskere Ashoka, ${ }^{1, \dagger}$ Pichandi Ashokkumar, ${ }^{1, \dagger}$ Yuriy P. Kovtun, ${ }^{2}$ Andrey S. Klymchenko ${ }^{1 * *}$

${ }^{1}$ Laboratoire de Bioimagerie et Pathologies, UMR 7021 CNRS, Faculté de Pharmacie, Université de Strasbourg, Strasbourg CS 60024, France

${ }^{2}$ Institute of Organic Chemistry, National Academy of Sciences of Ukraine, 5 Murmanska Street, 02094 Kyiv, Ukraine

$\dagger$ These co-authors contributed equally.

*Corresponding author: andrey.klymchenko@unistra.fr 


\begin{abstract}
Can polarity-sensitive fluorescent dyes monitor the response of live cells to fundamental stress conditions, such as deprivation from nutrition and oxidative stress? To address this question, we developed a push-pull dioxaborine probe (DXB-NIR) for biomembranes and lipid droplets featuring strong solvatochromism in far-red to near-infrared region, high fluorescence brightness, photostability and two-photon absorption cross section, reaching $13800 \mathrm{GM}$ at $925 \mathrm{~nm}$. In model membranes, DXBNIR exhibits unprecedented $80-\mathrm{nm}$ shift between liquid ordered and disordered membrane phases, allowing robust imaging of separated membrane microdomains. Two-color imaging of live cells with DXB-NIR enables polarity mapping in plasma membranes, endoplasmic reticulum and lipid droplets, which reveals that starvation and oxidative stress produce increase in the local polarity, and this change is different for each of the studied cell compartments. Thus, by pushing the limits of existing solvatochromic dyes, we introduce a concept of polarity mapping for monitoring the response of cells to stress.
\end{abstract}

\title{
TOC entry
}

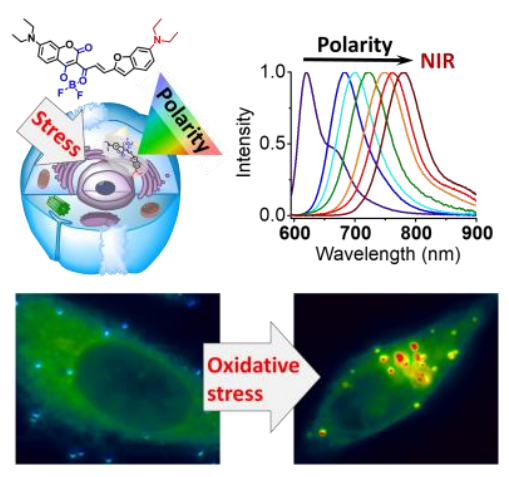

We developed a solvatochromic near-infrared dye based on push-pull dioxaborine and introduced a polarity mapping concept for monitoring cell stress. 
The response of the live cells to stress such as deprivation from nutrition (starvation) and oxidative stress is essential for cell survival. ${ }^{1,2}$ To monitor the cellular stress, an intensive research has been realized focusing on new fluorescent probes for detection of reactive oxygen species (ROS), ${ }^{3,4}$ such as hydrogen peroxide,${ }^{5}$ peroxyl ${ }^{6}$ peroxynitrite ${ }^{7}$ hypochlorite, ${ }^{8}$ etc. An attractive unexplored approach that does not require direct chemical reaction of the probe with ROS of interest is to monitor cellular stress through biophysical properties of biomembranes and lipid droplets. Starvation can significantly alter cell metabolism, favoring degradation of certain types of lipids. ${ }^{9}$ On the other hand, oxidative stress, ${ }^{10}$ can induce lipid peroxidation ${ }^{11}$, leading to dramatic alternation in the lipid organization.

The emerging powerful tools for deciphering lipid organization in live cells are so-called environmentsensitive fluorescent probes, ${ }^{12}$ sensitive to viscosity, lipid order and polarity. Dyes that undergo fast nonradiative deactivation through internal rotation, so-called molecular rotors, enable imaging membrane viscosity, which reflects lipid packing and mechanical properties of membranes. ${ }^{13,14}$ Fluorophores that undergo ground-state planarization in highly ordered membrane phases, so called flippers, were recently proposed to characterize lipid order and membrane tension in biomembranes ${ }^{15,16}$. Both molecular rotors and flippers were successfully used to monitor mechanical stress in live cells. ${ }^{13,15}$ Finally, solvatochromic dyes, ${ }^{17}$ push-pull fluorophores that undergo excited state charge transfer, can directly probe polarity of lipid structures ${ }^{12}$. Polarity is a particularly important parameter, because it is remarkably low for all lipid structures compared to those of proteins, nucleic acids and carbohydrates. Polarity-dependent shift in the emission spectrum of solvatochromic dyes can specifically describe lipid organization, in particular, lipid order. Indeed, solvatochromic membrane probes, such as Laurdan, ${ }^{18} \mathrm{NR} 12 \mathrm{~S},{ }^{19} \mathrm{di}-4-\mathrm{ANEPPDHQ},{ }^{20} \mathrm{etc}$., can distinguish by their emission color between liquid ordered (Lo) and liquid disordered (Ld) phases, which model formation of microdomains (so-called) rafts at the cell plasma membranes. ${ }^{21,22}$ Tightly packed lipids of Lo phase expel water and limit dipolar relations in the bilayer leading to low-polar environment recognized by these dyes. ${ }^{23}$ Moreover, solvatochromic dyes identify lipid droplets as highly apolar entities, ${ }^{24}$ because their core hosts the most lipophilic molecules of the cell. ${ }^{25,26}$ However, to date, solvatochromic dyes has not been reported to detect subtle changes in the lipid organization in cells under starvation and oxidative stress. The reasons are limited sensitivity of these probes to biophysical properties and their limited brightness, which do not allow analysis of their color response with sufficient contrast. The primary example is Laurdan, showing relatively good sensitivity to phase changes, ${ }^{18}$ but its brightness and photostability are limited ${ }^{24}$ and it operates in UV-visible spectral region. Nile Red (Fig. 1a), on the other hand, is brighter and it operates in suitable green-red region, which made it useful tool for imaging polarity in cells with nanoscopic resolution. ${ }^{27}$ However, its environment-sensitivity is weaker and it is 2-3-fold less bright than classical dyes, such as rhodamine or cyanines. ${ }^{12}$ In the last decade, multiple efforts have been done to improve these fluorophores, resulting in different push-pull fluorophores, based on fluorene, ${ }^{28}$ pyrene, ${ }^{29}$ DCDHF family, ${ }^{30}$ diphenylamino derivatives, ${ }^{31,32}$ phenylfurane, ${ }^{33} \mathrm{P}=\mathrm{O}-\mathrm{Rhodols}^{34}$, etc and some of them were successfully applied to study biomembranes ${ }^{24,35,36}$ and lipid droplets. ${ }^{24,31,32}$ Nevertheless, so far it remains a challenge to obtain a probe combining high solvatochromism, brightness, response to lipid order and operation in the far red to near infrared (NIR) region. The latter has multiple advantages, such as (i) low photo-toxicity and auto-fluorescence; (ii) color complementarity with commonly used fluorescent proteins; and (iii) deep tissue penetration in eventual small animal imaging. Boron-bridged pi-conjugated systems constitute a promising scaffold for highly emissive fluorophores. ${ }^{37}$ In particular, dioxaborine unit was successfully used to design cyanine or 
merocyanine-type dyes, ${ }^{38-40}$ featuring high brightness and photostability. Push-pull dioxaborines are much less explored, and their solvatochromism is limited. ${ }^{38,41}$

In this work, we have developed a solvatochromic dye based on push-pull dioxaborine (DXB-NIR, Fig. 1b), exhibiting NIR emission, high brightness and photo-stability as well as unprecedented $80 \mathrm{~nm}$ band shift in response to change in the lipid order. By changing its emission color, this probe provides the first evidence that starvation and oxidative stress increase significantly the local polarity in the intracellular membranes and in the lipid droplets. The developed probe proposes a new strategy to study response of the cell to the external stress.

Previously reported push-pull dioxaborine derivative showed limited solvatochromism, associated with molecular rotor properties. ${ }^{41}$ To enhance the push-pull character and to minimize rotational deactivation, we replaced phenyl with benzofurayl moiety, which was shown earlier to improve optical properties of push-pull 3-methoxychromones. ${ }^{42}$ The target dye DXB-NIR was obtained by Knoevenagel condensation of benzofuran aldehyde with corresponding dioxaborine-ketone (Fig. 1b and Scheme S1). Newly synthesized compound was characterized by standard analytical methods (Figs. S1-S9).

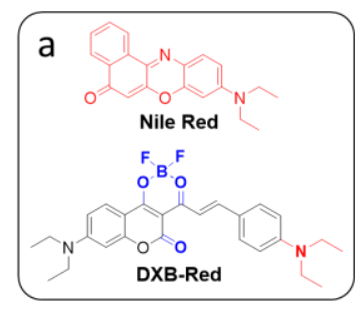

C

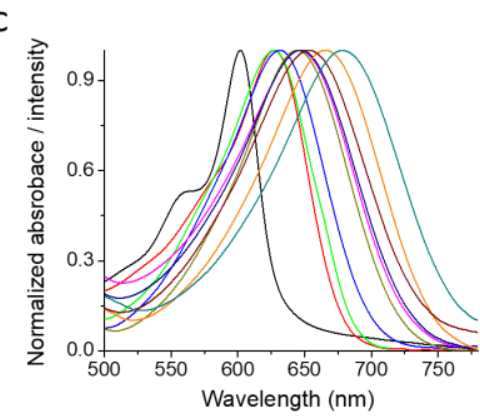

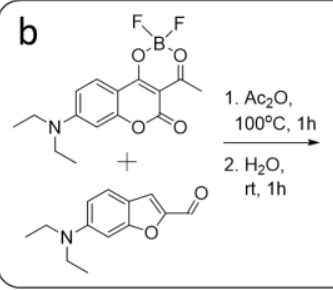

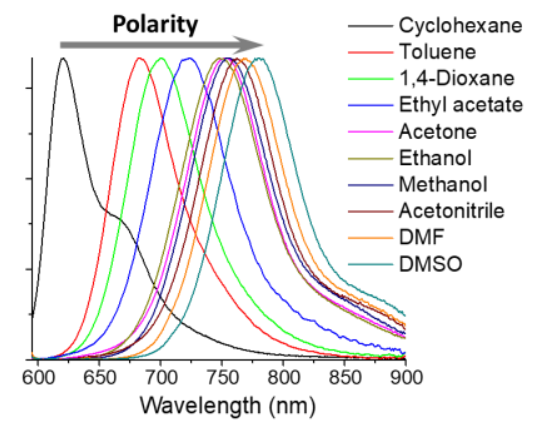

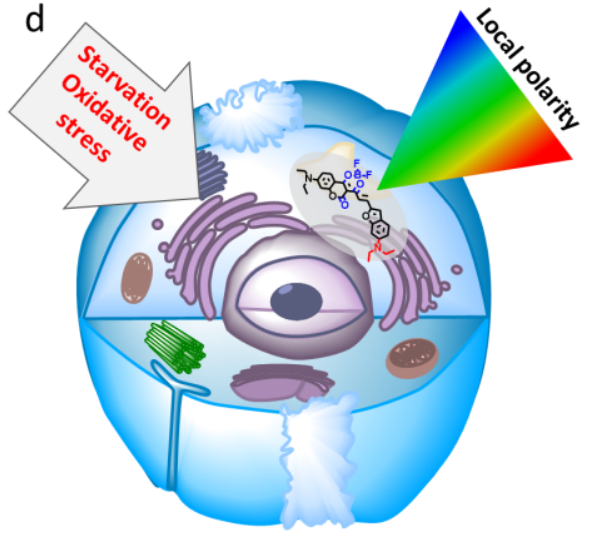

Fig. 1. (a) Previously reported push-pull dyes Nile Red and DXB-Red. (b) Synthesis of DXB-NIR. (c) normalized absorption and emission spectra of DXB-NIR (200 nM) in the organic solvents of varying polarity. For the fluorescence measurements DXB-NIR was excited at $580 \mathrm{~nm}$. (d) Scheme showing that DXB-NIR probe is expected to provide color response to local polarity changes in live cell at different stress conditions.

The absorption spectra of DXB-NIR in organic solvents cover red to NIR region (602-678 nm, Fig. 1c and Table 1). These values are red shifted by 95-120 nm with respect to Nile Red and by 60-70 $\mathrm{nm}$ with respect to the previously reported DXB-Red (Fig. 1a). ${ }^{41}$ The latter is the result of extended fluorophore conjugation. On increase in solvent polarity from non-polar cyclohexane to polar DMSO, the absorption spectra shifted from 602 to $678 \mathrm{~nm}$, respectively, thus showing positive solvatochromism. Remarkably, 
DXB-NIR showed $~ 2$-fold higher molar extinction coefficients in most of the studied solvents (83000$128000 \mathrm{M}^{-1} \mathrm{~cm}^{-1}$ ) compared to Nile Red (Table S1).

The emission spectra of DXB-NIR exhibited strong positive solvatochromism, as its maximum red shifted from $622 \mathrm{~nm}$ to $778 \mathrm{~nm}$ upon changing the solvent from apolar cyclohexane to polar DMSO (Fig. 1c, Table 1). This observation suggests a strong charge transfer character of the excited state of DXBNIR, in line with its push-pull structure. The emission maximum of Nile Red also showed red shift, but it was significantly smaller, namely from 528 till $636 \mathrm{~nm}$, respectively (Fig. S10). Similar to absorption spectra, the emission spectra of Nile Red in protic solvents showed red shifts compared to polar aprotic solvents, whereas for DXB-NIR they were significantly blue shifted (Table 1). These drastic differences can be due to the fact that in the case of DXB-NIR, the carbonyl lone pairs are involved in the complexation with boron, which should drastically decrease capacity of this carbonyl to form $\mathrm{H}$-bonds with protic solvents.

Table 1. Spectroscopic properties of Nile Red and DXB-NIR in large unilamellar vesicles (LUVs) of different compositions. ${ }^{\mathrm{a}}$

\begin{tabular}{|c|c|c|c|c|c|c|c|}
\hline & & \multicolumn{3}{|c|}{ Nile Red } & \multicolumn{3}{|c|}{ DXB-NIR } \\
\hline Solvent/LUVs & $\varepsilon$ & $\lambda_{\text {abs, max }}(\mathrm{nm})$ & $\lambda_{\text {em, max }}(\mathrm{nm})$ & $\begin{array}{c}\text { QY } \\
(\%)\end{array}$ & $\lambda_{\text {abs, max }}(\mathrm{nm})$ & $\lambda_{\text {em, max }}(\mathrm{nm})$ & $\begin{array}{c}\text { QY } \\
(\%)\end{array}$ \\
\hline Cyclohexane & 2.017 & 489 & 528 & 49 & 602 & 622 & 93 \\
\hline Toluene & 2.374 & 509 & 571 & 68 & 627 & 687 & 81 \\
\hline 1,4-Dioxane & 2.210 & 517 & 581 & 91 & 627 & 699 & 71 \\
\hline Ethyl acetate & 5.987 & 521 & 593 & 87 & 631 & 720 & 22 \\
\hline Acetone & 20.493 & 532 & 615 & 75 & 646 & 751 & 19 \\
\hline Ethanol & 24.852 & 550 & 636 & 52 & 645 & 748 & 18 \\
\hline Methanol & 32.613 & 553 & 640 & 38 & 647 & 754 & 3 \\
\hline Acetonitrile & 35.688 & 533 & 621 & 82 & 654 & 760 & 11 \\
\hline DMF & 37.219 & 544 & 626 & 63 & 666 & 769 & 9 \\
\hline DMSO & 46.826 & 554 & 636 & 46 & 678 & 778 & 3 \\
\hline Buffer & 78.355 & 593 & 667 & 5 & 585 & 721 & 0.03 \\
\hline DOPC & - & 545 & 641 & 41 & 641 & 715 & 35 \\
\hline DOPC/Chol & - & 550 & 635 & 45 & 632 & 711 & 44 \\
\hline SM/Chol & - & 538 & 589 & 36 & 617 & 635 & 27 \\
\hline Labrafac Oil & - & 531 & 590 & 55 & 628 & 701 & 39 \\
\hline
\end{tabular}

${ }^{\text {a }} \varepsilon$ is solvent dielectric constant; $\lambda$ abs, max and $\lambda$ em, max are absorption and emission maxima; QY (\%) is fluorescence quantum yield, measured using Nile Red in as reference (QY $=38 \%$ in methanol).

The fluorescence quantum yield of DXB-NIR showed high values in the apolar solvents and it gradually decreased in the polar solvents (Table 1). This behavior is typical for the dye undergoing strong excitedstate charge transfer, ${ }^{12,43}$ Remarkably, DXB-NIR was non-fluorescent in water, clearly due to the strong quenching phenomenon in this highly polar solvent. Moreover, as the absorption spectrum DXB-NIR in water is blue shifted compared to other solvents, we cannot exclude some dye aggregation accompanied by self-quenching. In a mixture of glycerol and methanol, absorption and emission spectra of DXB-NIR were found to be poorly sensitive to the viscosity (Fig. S11). Thus, in contrast to previously reported 
push-pull dioxaborine DXB-Red, ${ }^{41}$ DXB-NIR does not exhibit molecular rotor properties, probably because of its longer conjugation that ensures lower energy of its excited state and minimizes quenching through intramolecular rotation. Overall, the results suggest that DXB-NIR is promising as a fluorogenic and solvatochromic probe for polarity sensing in the apolar compartments of the cells such as biomembranes and lipid droplets.

Finally, two-photon (2P) absorption cross-section of DXB-NIR was evaluated, because this property is important in two-photon microscopy, a powerful tool in bioimaging. ${ }^{44}$ In three solvents, the intensity showed quadratic dependence on the 2P excitation power (Fig. S12) and the maximum of 2P absorption bands centered around $930 \mathrm{~nm}$ (Fig. S13). The 2P absorption values varied significantly with solvent, being the highest for dichloromethane (13800 GM) and the lowest for toluene (3240 GM). Such influence of solvent dielectric constant is known for push-pull dyes that increase their charge transfer character in more polar media. ${ }^{45}$ These values are $\sim 10$-fold higher than that of Nile Red. ${ }^{19}$ Remarkably, the obtained value of $13800 \mathrm{GM}$ at $925 \mathrm{~nm}$ for DXB-NIR in dichloromethane is among the largest reported to date for organic dyes and can only be compared to that of asymmetrical merocyanine-type dioxaborines. ${ }^{40,46}$ The remarkable $2 \mathrm{P}$ efficiency of our push-pull dioxaborine is probably linked to strong intramolecular charge transfer (ICT) character of this dye, which is known to be a key factor in the $2 \mathrm{P}$ absorption. ${ }^{44}$

We further evaluated the fluorescence response of DXB-NIR and Nile Red in large unilamellar vesicles (LUVs) of various lipid compositions. Binding to dioleoylphosphatidylcholine (DOPC) LUVs was accompanied by $~ 1000$-fold enhancement in the fluorescence quantum yield: $27-44 \%$ in LUVs vs $0.03 \%$ in water (Table 1). The fluorogenic response of DXB-NIR was significantly larger compared to Nile Red, which had non-negligible emission in aqueous media (5\%). Moreover, the absorption and emission maxima of DXB-NIR were 50-100 nm red shifted with respect to Nile Red (Table 1). Generally, solvatochromic dyes suffer from poor photostability. ${ }^{12}$ Moreover, shifting dyes towards NIR leads to decrease in their photostability. ${ }^{47}$ Remarkably, despite NIR emission of DXB-NIR in DOPC vesicles with maximum at $715 \mathrm{~nm}$, which was $74 \mathrm{~nm}$ red shifted compared to Nile Red, DXB-NIR showed a bit higher photostability than Nile Red (Fig. S14). This high photostability of DXB-NIR could be explained in part by the presence of the boron bridge, which should minimize excited state isomerization processes. $^{37,41}$ 

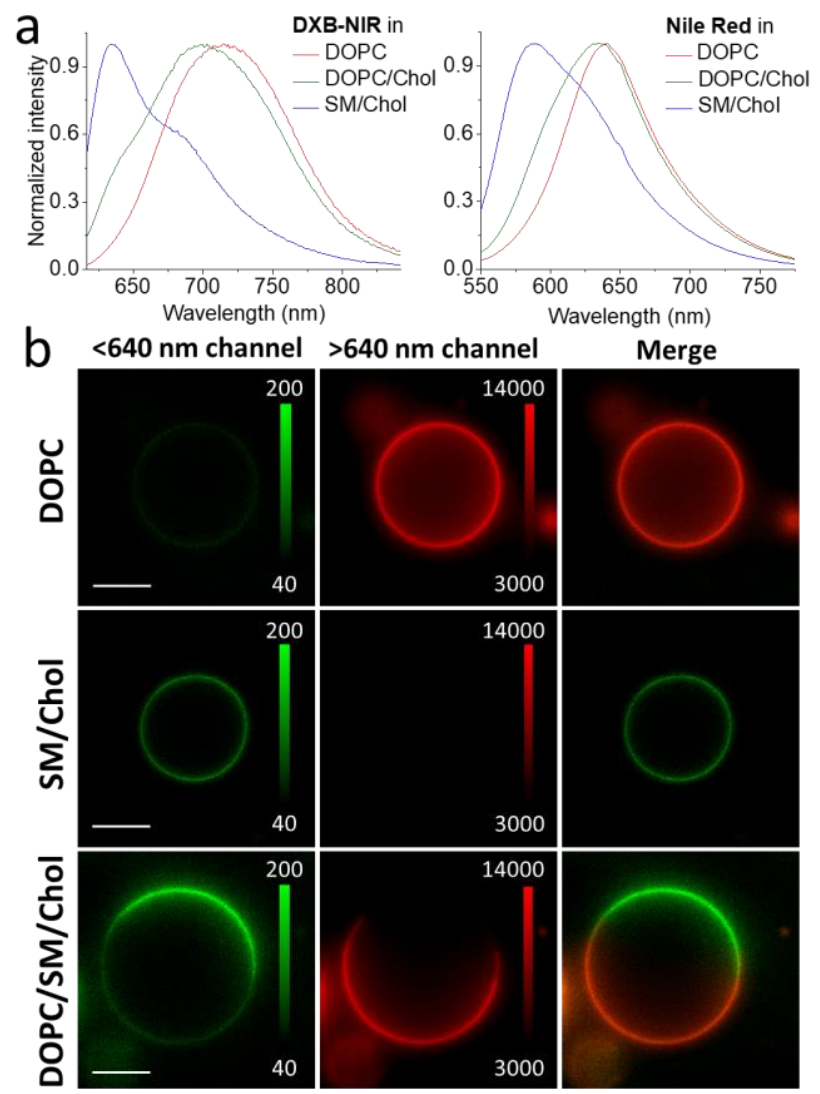

Fig. 2. Evaluation of DXB-NIR in model membranes. (a) Normalized emission spectra of Nile Red and DXB-NIR (200 $\mathrm{nM}$ ) in large unilamellar vesicles of various composition. For the emission measurements, Nile Red was excited at $530 \mathrm{~nm}$ and DXB-NIR was excited at $580 \mathrm{~nm}$. (b) Two-color fluorescence microscopy imaging of $\mathrm{L}_{d}, \mathrm{~L}_{0}$, and mixed $\mathrm{L}_{\mathrm{o}} / \mathrm{L}_{\mathrm{d}}$ phases in GUVs stained with DXB-NIR: far red channel $(<640 \mathrm{~nm})$, NIR channel $(>640 \mathrm{~nm})$ and merge of the two channels. GUVs were composed of DOPC $\left(\mathrm{L}_{\mathrm{d}}\right)$, SM/Chol (2/1) $\left(\mathrm{L}_{\mathrm{o}}\right)$, and DOPC/SM/Chol (1/1/0.5) ( $\mathrm{L}_{\mathrm{o}} / \mathrm{L}_{\mathrm{d}}$ mixture). The intensity scales are indicated for each channel. Excitation wavelength was $550 \mathrm{~nm}$. Scale bar, $10 \mu \mathrm{m}$.

The emission spectrum of DXB-NIR showed strong dependence on the lipid order in LUVs (Fig. 2a). In Lo phase of LUVs composed of sphingomyelin (SM) and cholesterol (Chol), the emission spectrum was centered at $635 \mathrm{~nm}$, which was significantly blue shifted compared to Ld vesicles made of DOPC or DOPC/Chol (Fig. 2a and Table 1). Similarly to Nile Red, ${ }^{19}$ DXB-NIR reports much lower polarity in Lo vs Ld phases, because of much tighter lipid packing in the former. Remarkably, the emission shifted by $80 \mathrm{~nm}$ from Ld to Lo phase, which is much higher than that of the Nile Red (52 nm). To the best of our knowledge, this 80-nm shift is the largest ever reported for a solvatochromic dye and can only be compared to mechanophores that can strongly shift their excitation ${ }^{16}$ or emission ${ }^{48}$ spectra in response to planarization in lipid bilayers of solid ordered (gel) phase.

Next, we performed fluorescence microscopy studies using giant unilamellar vesicles (GUVs) in order to visualize Lo and Ld phases. To image the spectral response of DXB-NIR, the signal was split into far 
red $(<640 \mathrm{~nm})$ and NIR $(>640 \mathrm{~nm})$ channels. GUVs presenting Ld phase (DOPC) showed a strong emission of DXB-NIR in the $>640 \mathrm{~nm}$ channel, whereas a negligible emission was observed in the $<640$ $\mathrm{nm}$ channel (Fig. 2b). Remarkably, in the case of GUVs presenting Lo phase (SM/Chol), the emission was mainly observed in the <640 channel (Fig. 2b). This impressive color switching is clearly due to strong (80-nm) band shift displayed by this dye when pure Lo and Ld phases are compared. Importantly, in the ternary mixture composed of both Lo and Ld phases (DOPC/SM/Chol) distinct emission was observed in the <640 and >640 channels and in the overlay image, so that Lo and Ld domains were easily distinguishable (Fig. 2b). For comparison, Nile Red based probe (NR12S) showed less clear color change in GUVs, so that the ratiometric analysis was required to distinguish Lo and Ld phases. ${ }^{19}$ Overall, DXBNIR displays remarkable sensitivity to lipid order and could be of interest to detect subtle changes in the lipid organization in eukaryotic cells.

We further characterized the staining of the cells with DXB-NIR using fluorescence microscopy. Live cells incubated with DXB-NIR displayed intense fluorescence in different cellular compartments. To understand better dye bio-distribution, we localized its fluorescence with different cellular markers (Fig. 3a): (i) BODIPY 493/503 for lipid droplets, (ii) ER tracker green for endoplasmic reticulum and (iii) F2N12SM ${ }^{49}$ for plasma membranes (PM). First, the dotted fluorescence of DXB-NIR inside the cells colocalized well with BODIPY 493/503 (Fig. 3a), suggesting that it originates from LDs. Second, the continuous fluorescence inside the cells corresponded well to the staining with ER tracker (Fig. 3a), indicating that the DXB-NIR also distributed in the membranes of endoplasmic reticulum. Finally, nonnegligible fluorescence of DXB-NIR was also observed at the cell contour (Fig. 3a and S15), which corresponded to cell PM, in line with the F2N12SM staining. We can conclude that, owing to its lipophilic nature, DXB-NIR distributes all over different lipid structures of the cells, which enabled us to map distribution polarity all over these structures. To this end, we split the emission of the dye at 640 $\mathrm{nm}$, as we did for GUVs and studied intact live cells stained exclusively with DXB-NIR. Both <640 and $>640 \mathrm{~nm}$ channels, presented in green and red, respectively, exhibited considerable fluorescence, and the merge of these two channels revealed variation of pseudo-color throughout the cell (Fig. 3b), suggesting uneven polarity distribution in the lipid compartments. To provide a quantitative information, we analyzed the ratio of NIR to far red channels, $\mathrm{I}(>640) / \mathrm{I}(<640)$ in cells as well as in different model media: apolar oil of medium chain triglycerides (Labrafac) modeling LDs and lipid vesicles (LUVs) composed of SM/Chol and DOPC, modeling Lo and Ld phases in biomembranes. The ratio images of cells (Fig. 3b, c, e) confirmed the heterogeneity within different cellular compartments, observed in the merged images. Remarkably, magenta pseudo-color observed for LDs in cells corresponded to that observed for DXB-NIR in apolar oil (Fig. 3b and c). Blue pseudo-color of PM matched well to the $\mathrm{SM} / \mathrm{Chol}$, which confirmed that cell plasma membranes rich in SM and cholesterol present highly ordered state close to Lo. ${ }^{19,24,50}$ Finally, green-yellow color corresponding to intracellular membranes showed significantly higher polarity, compared to LDs and PM. The quantitative ratio analysis confirmed this observation, showing that polarity in PM, intracellular membranes (IM) and LDs corresponded to that in SM/Chol LUVs, DOPC LUVs and Labrafac oil (Fig. 3d). The observed higher polarity of intracellular membranes compared to PM is in agreement with previous reports ${ }^{24}$ and reflects much lower content in cholesterol and SM in the intracellular membranes. ${ }^{51}$ Thus, by changing its emission color between far-red and NIR regions, DXB-NIR could clearly distinguish polarity of different cellular lipid compartments. These results corroborated with two-photon excited imaging of HeLa cells stained 
with DXB-NIR, revealing uneven polarity distribution in the two-color merge and ratio images, featuring a nearly zero background (Fig. S16). Even though DXB-NIR shows solvent polarity dependent 2P absorption cross-section, it is high enough (3240 - $13800 \mathrm{GM})$ in the whole polarity range to ensure cell polarity mapping. This result suggests that DXB-NIR is also compatible with $2 \mathrm{P}$ imaging.

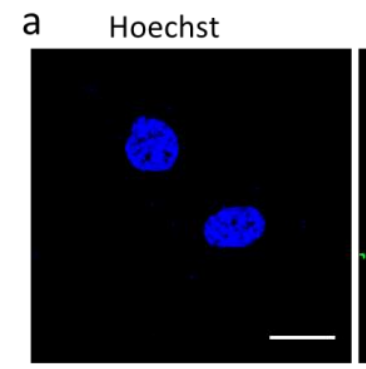

F2N12SM

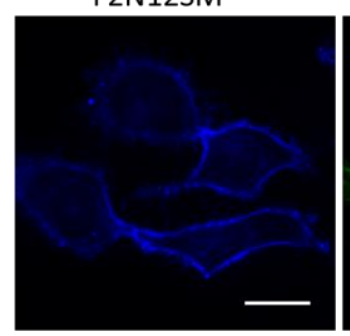

b $\quad<640 \mathrm{~nm}$ channel
BODIPY

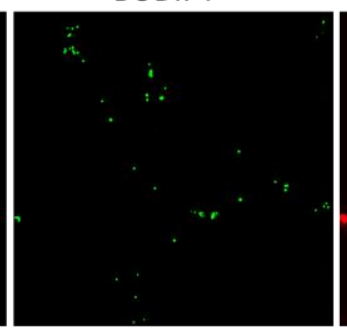

ER tracker

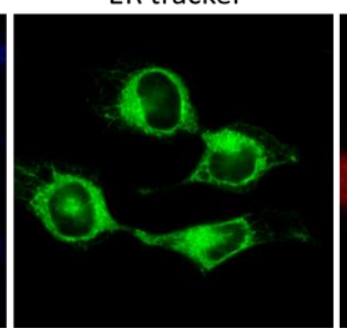

DXB-NIR

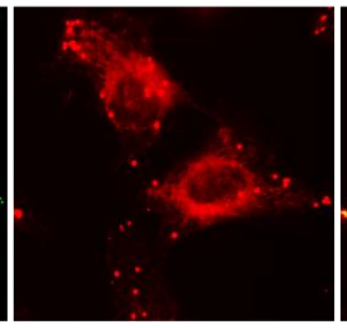

DXB-NIR

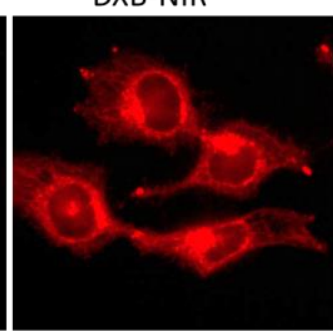

Merge

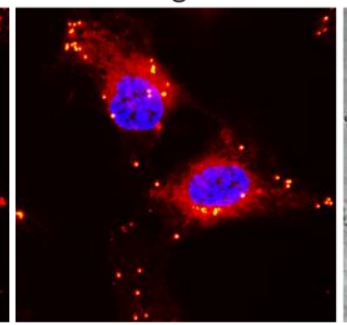

Merge

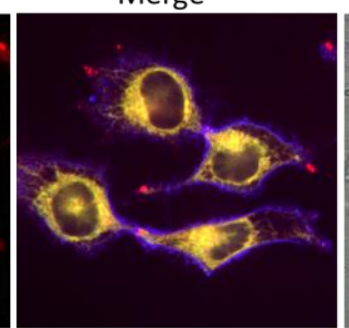

Brightfield

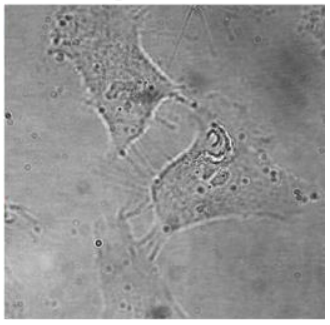

Brightfield

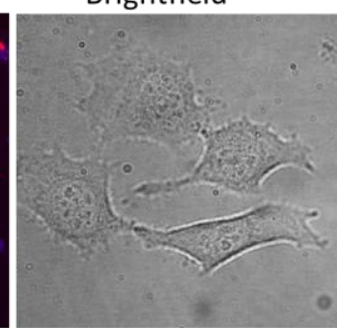

$\mathrm{I}_{>640} / \mathrm{I}_{<640}$ ratio
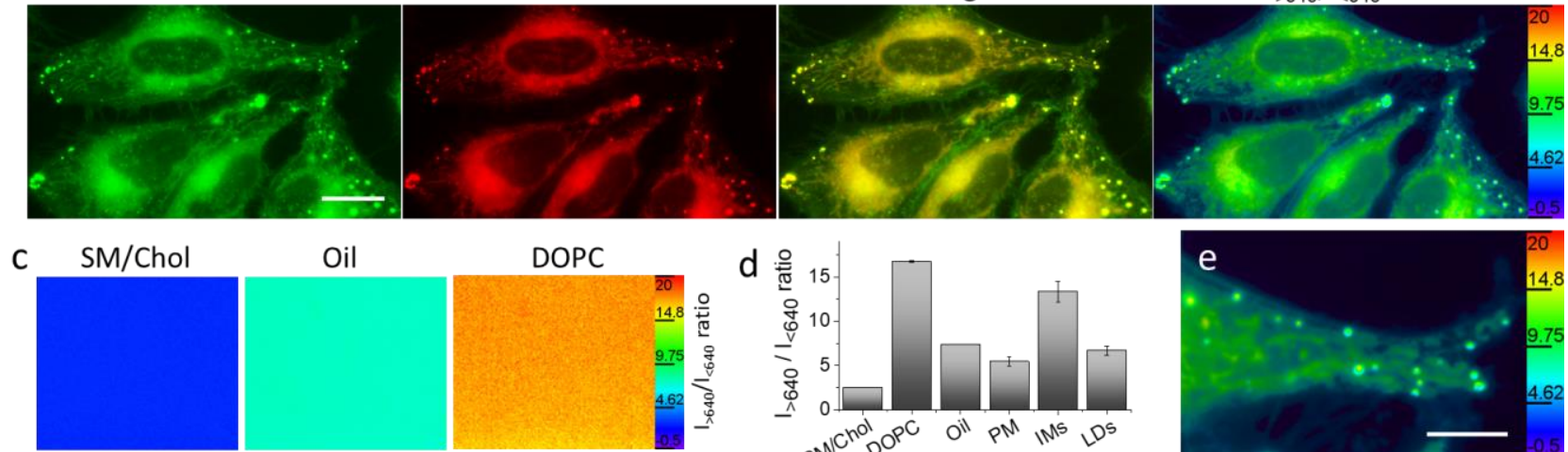

Oil

DOPC
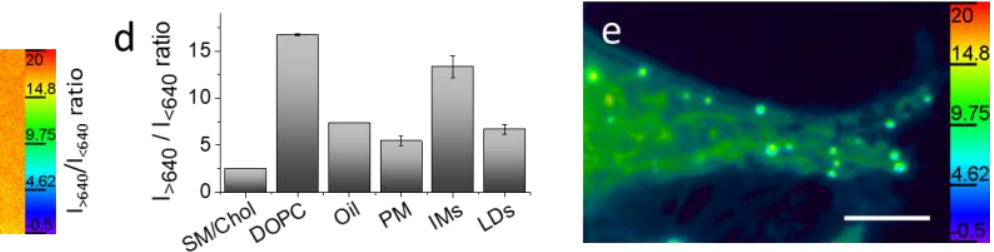

Fig. 3. Imaging of HeLa cells with DXB-NIR. (a) Spinning-disk fluorescence microscopy of intracellular distribution of DXB-NIR Probe (200 nM, incubated with CD in OptiMEM for 30 min at $\left.20{ }^{\circ} \mathrm{C}\right)$ costained with membrane probe F2N12SM (100 nM, in HBSS for $7 \mathrm{~min}$ at 20 $\mathrm{C}$ ), ER tracker Green (1 $\mathrm{mM}$, incubated in OptiMEM for $30 \mathrm{~min}$ at $37^{\circ} \mathrm{C}$ ) and LDs marker BODIPY 493/503 (500 $\mathrm{nM}$, in OptiMEM for $5 \mathrm{~min}$ at $20^{\circ} \mathrm{C}$ ). Scale bar, $20 \mu \mathrm{m}$. (b) Two-color wide-field fluorescence imaging of DXBNIR in live cells: far red channel $(<640 \mathrm{~nm})$, NIR channel $(>640 \mathrm{~nm})$, merge of the two channels and the ratio of NIR to far red channels ( $\left.\mathrm{I}_{>640} / \mathrm{I}_{<640}\right)$. Scale bar, $10 \mu \mathrm{m}$. (c) Ratio $\left(\mathrm{I}_{>640} / \mathrm{I}_{<640}\right)$ images of DXB-NIR in LUVs composed of SM/Chol and DOPC and in Labrafac oil. Excitation wavelength was $550 \mathrm{~nm}(\mathrm{~b}, \mathrm{c})$. (d) NIR/far red intensity ratio $\left(\mathrm{I}_{>640} / \mathrm{I}_{<640}\right)$ in LUVs composed of SM/Chol and DOPC, Labrafac oil, and in different cell compartments: plasma membranes (PM), intracellular membranes (IMs) and lipid droplets (LDs), obtained from fluorescence images (b) and (c). (e) Zoomed ratio image (b); scale bar, 5 $\mu \mathrm{m}$. 
To generate a model stress condition that can drastically alter properties of biomembranes, we deprived cells from cholesterol using methyl- $\beta$-cyclodextrin $(\mathrm{M} \beta C \mathrm{CD})^{52}$ and imaged them in the two-color mode. Cholesterol extraction induced gradual increase in the relative intensity at the NIR emission channel (>640 $\mathrm{nm}$ ), indicating polarity increase and the decrease in the lipid order in different biomembranes (Fig. 4a, c, e). To better visualize changes at the two channels, ratiometric images were generated (Fig. $4 \mathrm{~b}, \mathrm{~d}, \mathrm{f})$. They confirm the observations in the merged images showing that the ratio NIR to far red increased with cholesterol depletion time. At 30 min depletion time, the relative intensity in the NIR channel increased significantly only for the intracellular membranes, which was confirmed by the analysis of the ratio changes (Fig. 5c). On the other hand, for $2 \mathrm{~h}$ of the depletion, the changes were already observed for all three lipid compartments studied (Fig. 4f, 5c). This result suggests that cholesterol depletion with $\mathrm{M} \beta \mathrm{CD}$ produces the fastest effect at the level of intracellular membranes, in line with an earlier report, where cholesterol depletion was shown to be much faster in ER than in PM. ${ }^{53}$ We could speculate that ER membranes could easily lose the small amount of cholesterol they contain, which seems not to be the case for LDs and PM. In case of PM, cholesterol is strongly associated with sphingomyelin, whereas in LDs cholesterol is present in the form of esters, which should be hydrolyzed before the removal. Nevertheless, $2 \mathrm{~h}$ of cholesterol extraction appears sufficient to produce strong changes in all studied lipid compartments. These results suggest that DXB-NIR is useful to study changes in cholesterol distribution throughout the cell.
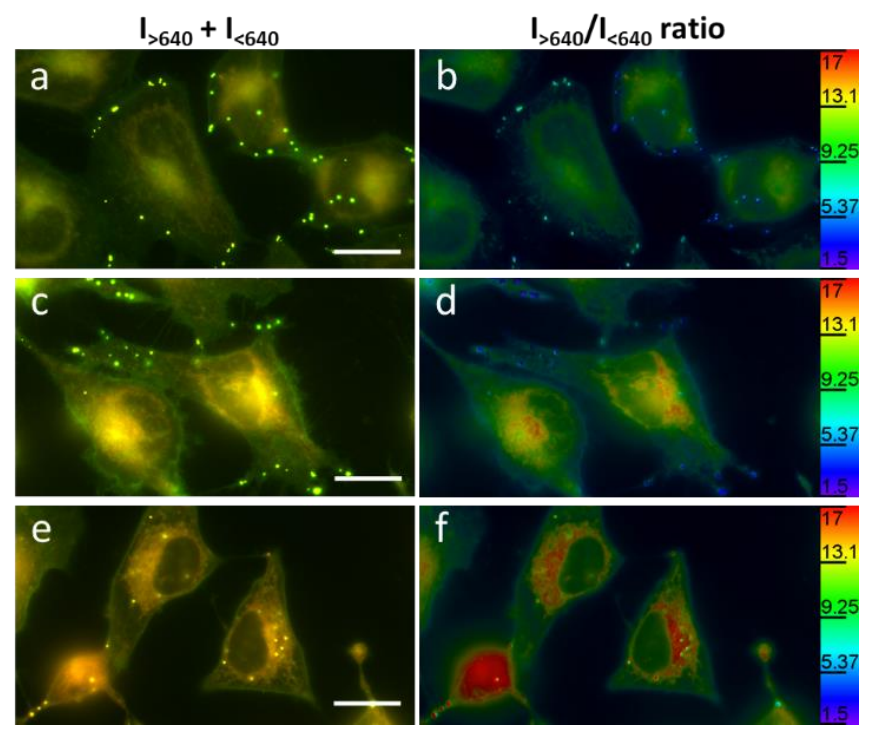

Fig. 4. Cholesterol depletion studies in HeLa cells stained with DXB-NIR (200 nM). (a,b) Intact cells $(a, b)$; cells treated with M MCD $(2 \mathrm{mg} / \mathrm{ml})$ for $30 \mathrm{~min}(\mathrm{c}, \mathrm{d})$ and $2 \mathrm{~h}(\mathrm{e}, \mathrm{f})$. (a,c,e)Merged images in far red (presented in green) and NIR (presented in red) channels. (b,d,f) Ratio images of NIR to far red channels $\left(\mathrm{I}_{>640} / \mathrm{I}_{6640}\right)$. A dichroic mirror at $640 \mathrm{~nm}$ was used to split the emission into the two channels. Excitation wavelength was $550 \mathrm{~nm}$. Scale bar, $10 \mu \mathrm{m}$. 
To evaluate the effect of starvation, HeLa cells were maintained under serum-free conditions for 3 and $24 \mathrm{~h}$ and then stained with DXB-NIR. The bright spots of DXB-NIR in starved cells ( $24 \mathrm{~h})$ colocalized well with the LDs marker BODIPY 493/503, which confirms the presence of LDs and their labeling by our probe even after starvation (Fig. S17). The two-color imaging suggested a gradual increase in the relative intensity of the NIR channel with the starvation time (Fig. 5a, S18). The shift of DXB-NIR emission towards NIR region in response to the starvation was in agreement with the analyzed probe ratiometric response in the intracellular membranes and LDs (Fig. 5c) and with the histogram of the NIR/far red ratio distribution over the cells (Fig. S19). This spectral response indicated that polarity at different lipid compartments of starved cells increased over time. During the starvation, the cells are expected to use lipids as the energy source for their survival, which in turn alter the lipid order and hence the polarity monitored by DXB-NIR. The changes were particularly well seen at the level of LDs and intracellular membranes (Fig. 5a, 5c). As starvation is known to cause consumption and lipolysis of lipid components of LDs, ${ }^{9,54}$ the hydrophobic core of LDs should shrink, which is probably detected by DXBNIR as the increase in their polarity. Moreover, previous studies showed that starvation can be a source of autophagy and oxidative stress, ${ }^{9,55}$ which may favor lipid peroxidation, ${ }^{11}$ including that in LDs, ${ }^{56}$ which could also explain the observed increase in the local polarity at the different lipid structures. Therefore, we further studied the effect of oxidative stress in cells by DXB-NIR.
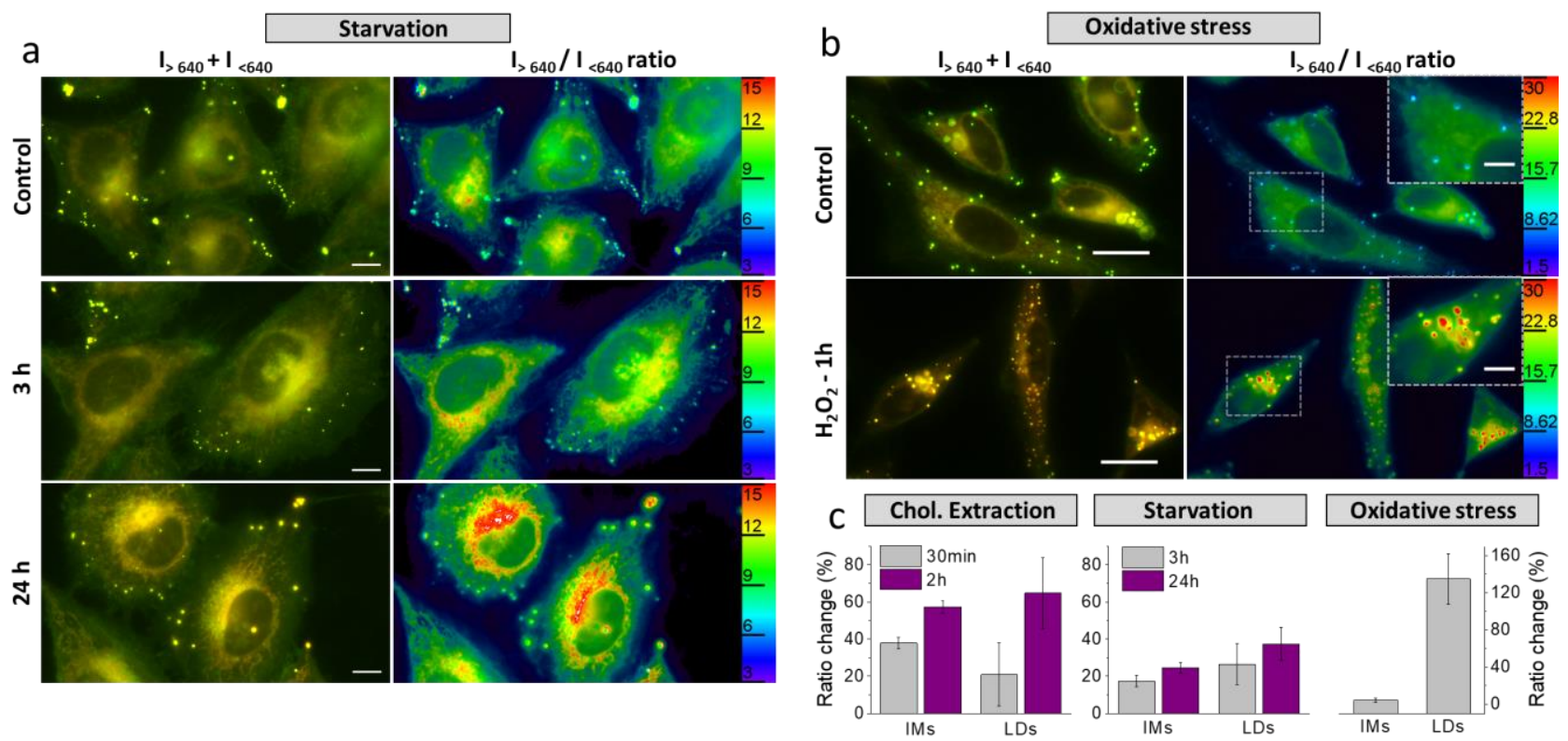

Fig. 5. Imaging HeLa cells under starvation and oxidative stress using DXB-NIR (200 nM): two-color merge (left) and ratio (right) images. (a) Intact cells and cells after 3 and $24 \mathrm{~h}$ starvation. (b) Intact cells and cells treated with $2 \mathrm{mM} \mathrm{H}_{2} \mathrm{O}_{2}$ for $1 \mathrm{~h}$. A dichroic mirror at $640 \mathrm{~nm}$ was used to split the emission into the two channels. Excitation wavelength was $550 \mathrm{~nm}$. Scale bar for main images (a,b), $10 \mu \mathrm{m}$; for insets, $3 \mu \mathrm{m}$. (c) Changes in the NIR/far red intensity ratio $\left(\left(\mathrm{R}-\mathrm{R}_{0}\right) / \mathrm{R}_{0} \times 100 \%\right.$, where $\mathrm{R}$ and $\mathrm{R}_{0}$ are the average intensity ratios of the stressed and intact cells, respectively) in the intracellular membranes (IMs) and lipid droplets (LDs) after cholesterol extraction, starvation and oxidative stress measured from corresponding fluorescence images. 
The exposure of cells to hydrogen peroxide $\left(\mathrm{H}_{2} \mathrm{O}_{2}\right)$ leads to oxidative stress, accompanied by lipid peroxidation $^{11}$ that should alter structure and properties of lipid-based cell compartments. Here, the cells treated with $\mathrm{H}_{2} \mathrm{O}_{2}(2 \mathrm{mM} \text { for } 1 \mathrm{~h})^{57}$ were further stained with DXB-NIR and imaged by two-color fluorescence microscopy. The obtained images suggested that $\mathrm{H}_{2} \mathrm{O}_{2}$ treatment induced drastic increase in the relative fluorescence signal at the NIR channel in cytoplasm and especially in LDs (Fig. 5b), which is already evident from the images with two channels merged (Fig. 5b, left). Analysis of the intensity ratio (Fig. 5b, right) further confirmed the drastic increase in the NIR emission of DXB-NIR in the $\mathrm{H}_{2} \mathrm{O}_{2-}$ treated cells. The effect is particularly strong at the level of LDs, where the NIR/far red ratio increased $>2$-fold from 8-10 (blue pseudo-color) to 22-25 (yellow-orange). The observation is confirmed by the analysis of the probe ratiometric response, showing that major changes are observed in LDs (Fig. 5c). It should be noted that after $\mathrm{H}_{2} \mathrm{O}_{2}$ treatment DXB-NIR colocalized well with LDs marker BODIPY 493/503, which also confirmed the presence of LDs and their labelling by DXB-NIR after the oxidative stress (Fig. S20). To the best of our knowledge, the direct effect of oxidative stress on LDs polarity is reported for the first time. This could imply that LDs, rich in cholesterol and unsaturated lipids, are prone to oxidize rapidly under the condition of oxidative stress. Moreover, LDs has been recently shown to play protective antioxidant role in different cell types, ${ }^{58,59}$ which suggest that during oxidative stress LDs may undergo oxidation faster than other components. On the other hand, the cell plasma membrane and other intracellular membranes were less affected at this step, probably because of multiple anti-oxidant defense mechanisms. Thus, DXB-NIR can monitor oxidative stress in cells, but, unlike classical probes of oxidative stress that directly react with $\operatorname{ROS},{ }^{3}$ its response is indirect, being linked to changes in the local polarity in lipid compartments.

In conclusion, we have developed a push-pull dioxaborine probe, DXB-NIR, showing strong solvatochromism in the emission changing from the far red to NIR region, high fluorescence quantum yield, photostability and molar extinction coefficient as well as outstanding two-photon absorption cross section at $930 \mathrm{~nm}$. In model lipid membranes it showed unprecedented solvatochromic shift of $80 \mathrm{~nm}$ when liquid ordered and disordered membrane phases are compared, which enabled high-contrast imaging of these phases in giant vesicles. Importantly, two-color ratiometric imaging of cells in far-red and NIR regions using DXB-NIR dye allowed polarity mapping of different lipid structures, such as plasma membranes, endoplasmic reticulum and lipid droplets. The polarity mapping with DXB-NIR in live cells was also achieved using two-photon microscopy. Moreover, DXB-NIR probe revealed that different stress conditions, such as cholesterol extraction, starvation and oxidative stress produced drastic increase in local polarity, which was characteristic for each lipid compartment. Indeed, cholesterol extraction had a strong and rapid effect on endoplasmic reticulum, whereas starvation and oxidative stress affected strongly polarity of lipid droplets with less pronounced changes in other lipid structures. Thus, based on a powerful solvatochromic dye, we propose a concept of polarity mapping of lipid compartments of cells for monitoring their response to starvation and oxidative stress, which are in the center of variety of pathologies.

\section{ASSOCIATED CONTENT}

Supporting Information. Materials and Methods section, additional spectroscopy and microscopy data. 


\section{ACKNOWLEDGMENT}

This work was supported by the European Research Council ERC Consolidator grant BrightSens 648528. AHA acknowledges Indo-French Centre for the Promotion of Advanced Research for Raman-Charpak fellowship.

\section{REFERENCES}

(1) Jeon, S. M.; Chandel, N. S.; Hay, N. AMPK Regulates NADPH Homeostasis to Promote Tumour Cell Survival During Energy Stress. Nature 2012, 485, 661-665.

(2) Boroughs, L. K.; DeBerardinis, R. J. Metabolic Pathways Promoting Cancer Cell Survival and Growth. Nat. Cell Biol. 2015, 17, 351-359.

(3) Chen, X. Q.; Wang, F.; Hyun, J. Y.; Wei, T. W.; Qiang, J.; Ren, X. T.; Shin, I.; Yoon, J. Recent Progress in The Development of Fluorescent, Luminescent and Colorimetric Probes for Detection of Reactive Oxygen and Nitrogen Species. Chem. Soc. Rev. 2016, 45, 2976-3016.

(4) Kaur, A.; Kolanowski, J. L.; New, E. J. Reversible Fluorescent Probes for Biological Redox States. Angew. Chem. Int. Ed. 2016, 55, 1602-1613.

(5) Dickinson, B. C.; Huynh, C.; Chang, C. J. A Palette of Fluorescent Probes with Varying Emission Colors for Imaging Hydrogen Peroxide Signaling in Living Cells. J. Am. Chem. Soc. 2010, 132, 5906-5915.

(6) Oleynik, P.; Ishihara, Y.; Cosa, G. Design and Synthesis of a Bodipy-Alpha-Tocopherol Adduct for Use as An Off/On Fluorescent Antioxidant Indicator. J. Am. Chem. Soc. 2007, 129, 1842-1843.

(7) Cheng, D.; Pan, Y.; Wang, L.; Zeng, Z. B.; Yuan, L.; Zhang, X. B.; Chang, Y. T. Selective Visualization of the Endogenous Peroxynitrite in an Inflamed Mouse Model by a Mitochondria-Targetable Two-Photon Ratiometric Fluorescent Probe. J. Am. Chem. Soc. 2017, 139, 285-292.

(8) Xu, Q.; Heo, C. H.; Kim, G.; Lee, H. W.; Kim, H. M.; Yoon, J. Development of Imidazoline-2-Thiones Based Two-Photon Fluorescence Probes for Imaging Hypochlorite Generation in a Co-Culture System. Angew. Chem. Int. Ed. 2015, 54, 4890-4894.

(9) Velazquez, A. R.; Tatsuta, T.; Ghillebert, R.; Drescher, I.; Graef, M. Lipid Droplet-Mediated ER Homeostasis Regulates Autophagy and Cell Survival During Starvation. J. Cell Biol. 2016, 212, 621-631.

(10) Finkel, T.; Holbrook, N. J. Oxidants, Oxidative Stress and the Biology of Ageing. Nature 2000, 408, 239247.

(11) Yin, H. Y.; Xu, L. B.; Porter, N. A. Free Radical Lipid Peroxidation: Mechanisms and Analysis. Chem. Rev. 2011, 111, 5944-5972.

(12) Klymchenko, A. S. Solvatochromic and Fluorogenic Dyes as Environment-Sensitive Probes: Design and Biological Applications. Accounts of Chemical Research 2017, 50, 366-375.

(13) Chambers, J. E.; Kubankova, M.; Huber, R. G.; Lopez-Duarte, I.; Avezov, E.; Bond, P. J.; Marciniak, S. J.; Kuimova, M. K. An Optical Technique for Mapping Microviscosity Dynamics in Cellular Organelles. ACS Nano 2018, 12, 4398-4407.

(14) Kuimova, M. K.; Yahioglu, G.; Levitt, J. A.; Suhling, K. Molecular Rotor Measures Viscosity of Live Cells via Fluorescence Lifetime Imaging. J. Am. Chem. Soc. 2008, 130, 6672-+.

(15) Colom, A.; Derivery, E.; Soleimanpour, S.; Tomba, C.; Dal Molin, M.; Sakai, N.; Gonzalez-Gaitan, M.; Matile, S.; Roux, A. A Fluorescent Membrane Tension Probe. Nat. Chem. 2018, 10, 1118-1125.

(16) Dal Molin, M.; Verolet, Q.; Colom, A.; Letrun, R.; Derivery, E.; Gonzalez-Gaitan, M.; Vauthey, E.; Roux, A.; Sakai, N.; Matile, S. Fluorescent Flippers for Mechanosensitive Membrane Probes. J. Am. Chem. Soc. 2015, 137, 568-571.

(17) Reichardt, C. Solvatochromic Dyes as Solvent Polarity Indicators. Chem. Rev. 1994, 94, 2319-2358.

(18) Parasassi, T.; Krasnowska, E. K.; Bagatolli, L.; Gratton, E. LAURDAN and PRODAN as Polarity-Sensitive Fluorescent Membrane Probes. J. Fluoresc. 1998, 8, 365-373. 
(19) Kucherak, O. A.; Oncul, S.; Darwich, Z.; Yushchenko, D. A.; Arntz, Y.; Didier, P.; Mely, Y.; Klymchenko, A. S. Switchable Nile Red-Based Probe for Cholesterol and Lipid Order at the Outer Leaflet of Biomembranes. J. Am. Chem. Soc. 2010, 132, 4907-4916.

(20) Jin, L.; Millard, A. C.; Wuskell, J. P.; Dong, X. M.; Wu, D. Q.; Clark, H. A.; Loew, L. M. Characterization and Application of a New Optical Probe for Membrane Lipid Domains. Biophys. Journal 2006, 90, 2563-2575. Lingwood, D.; Simons, K. Lipid Rafts as a Membrane-Organizing Principle. Science 2010, 327, 46-50. Regulation and Roles of Lipid Rafts. Nat. Rev. Mol. Cell Biol. 2017, 18, 361.

Klymchenko, A. S.; Kreder, R. Fluorescent Probes for Lipid Rafts: From Model Membranes to Living Cells. Chem. Biol. 2014, 21, 97-113.

Niko, Y.; Didier, P.; Mely, Y.; Konishi, G.; Klymchenko, A. S. Bright and Photostable Push-Pull Pyrene Dye Visualizes Lipid Order Variation between Plasma and Intracellular Membranes. Sci. Rep. 2016, 6, 18870. Guo, Y.; Cordes, K. R.; Farese, R. V.; Walther, T. C. Lipid Droplets at a Glance. Journal of Cell Science 2009, 122, 749-752.

Martin, S.; Parton, R. G. Lipid Droplets: a Unified View of a Dynamic Organelle. Nat. Rev. Mol. Cell Biol. 2006, 7, 373-378.

Yan, R.; Moon, S.; Kenny, S. J.; Xu, K. Spectrally Resolved and Functional Super-resolution Microscopy via Ultrahigh-Throughput Single-Molecule Spectroscopy. Accounts Chem. Res. 2018, 51, 697-705.

Kucherak, O. A.; Didier, P.; Mely, Y.; Klymchenko, A. S. Fluorene Analogues of Prodan with Superior Fluorescence Brightness and Solvatochromism. J. Phys. Chem. Lett. 2010, 1, 616-620.

Niko, Y.; Kawauchi, S.; Konishi, G.-i. Solvatochromic Pyrene Analogues of Prodan Exhibiting Extremely High Fluorescence Quantum Yields in Apolar and Polar Solvents. Chem.-Eur. J. 2013, 19, 9760-9765.

Lord, S. J.; Conley, N. R.; Lee, H. L. D.; Nishimura, S. Y.; Pomerantz, A. K.; Willets, K. A.; Lu, Z. K.; Wang, H.; Liu, N.; Samuel, R.et al. DCDHF Fluorophores for Single-Molecule Imaging in Cells. ChemPhysChem 2009, $10,55-65$.

Jiang, M. J.; Gu, X. G.; Lam, J. W. Y.; Zhang, Y. L.; Kwok, R. T. K.; Wong, K. S.; Tang, B. Z. Two-Photon AlE Bio-Probe with Large Stokes Shift for Specific Imaging of Lipid Droplets. Chem. Sci. 2017, 8, 5440-5446.

Zheng, Z.; Zhang, T. F.; Liu, H. X.; Chen, Y. C.; Kwok, R. T. K.; Ma, C.; Zhang, P. F.; Sung, H. H. Y.; Williams, I. D.; Lam, J. W. Y.et al. Bright Near-Infrared Aggregation-Induced Emission Luminogens with Strong TwoPhoton Absorption, Excellent Organelle Specificity, and Efficient Photodynamic Therapy Potential. ACS Nano 2018, 12, 8145-8159.

Collot, M.; Bou, S.; Fam, T. K.; Richert, L.; Mély, Y.; Danglot, L.; Klymchenko, A. S. Probing Polarity and Heterogeneity of Lipid Droplets in Live Cells Using a Push-Pull Fluorophore. Analytical Chemistry 2019, 91, 1928-1935.

Grzybowski, M.; Taki, M.; Yamaguchi, S. Selective Conversion of P=O-Bridged Rhodamines into $\mathrm{P}=\mathrm{O}-$ Rhodols: Solvatochromic Near-Infrared Fluorophores. Chem.-Eur. J. 2017, 23, 13028-13032.

Shaya, J.; Collot, M.; Benailly, F.; Mahmoud, N.; Mely, Y.; Michel, B. Y.; Klymchenko, A. S.; Burger, A. Turnon Fluorene Push-Pull Probes with High Brightness and Photostability for Visualizing Lipid Order in Biomembranes. ACS Chem. Biol. 2017, 12, 3022-3030.

Manni, M. M.; Tiberti, M. L.; Pagnotta, S.; Barelli, H.; Gautier, R.; Antonny, B. Acyl chain asymmetry and polyunsaturation of brain phospho ipids facilitate membrane vesiculation without leakage. eLife 2018, 7, e34394.

Frath, D.; Massue, J.; Ulrich, G.; Ziessel, R. Luminescent Materials: Locking pi-Conjugated and Heterocyclic Ligands with Boron(III). Angew. Chem. Int. Ed. 2014, 53, 2290-2310.

Gerasov, A. O.; Shandura, M. P.; Kovtun, Y. P. Series of Polymethine Dyes Derived from 2,2-Difluoro1,3,2-(2h)-Dioxaborine Of 3-Acetyl-7-Diethylamino-4-Hydroxycoumarin. Dyes Pigm. 2008, 77, 598-607.

Gerasov, A. O.; Shandura, M. P.; Kovtun, Y. P. Polymethine Dyes Derived from the Boron Difluoride Complex of 3-Acetyl-5,7-Di(Pyrrolidin-1-YI)-4-Hydroxycoumarin. Dyes Pigm. 2008, 79, 252-258. 
(40) Collot, M.; Fam, T. K.; Ashokkumar, P.; Faklaris, O.; Galli, T.; Danglot, L.; Klymchenko, A. S. Ultrabright and Fluorogenic Probes for Multicolor Imaging and Tracking of Lipid Droplets in Cells and Tissues. J. Am. Chem. Soc. 2018, 140, 5401-5411.

(41) Karpenko, I. A.; Niko, Y.; Yakubovskyi, V. P.; Gerasov, A. O.; Bonnet, D.; Kovtun, Y. P.; Klymchenko, A. S. Push-Pull Dioxaborine as Fluorescent Molecular Rotor: Far-Red Fluorogenic Probe for Ligand-Receptor Interactions. J. Mater. Chem. C 2016, 4, 3002-3009.

(42) Kucherak, O. A.; Richert, L.; Mely, Y.; Klymchenko, A. S. Dipolar 3-Methoxychromones as Bright and Highly Solvatochromic Fluorescent Dyes. Phys. Chem. Chem. Phys. 2012, 14, 2292-2300.

(43) Grabowski, Z. R.; Rotkiewicz, K.; Rettig, W. Structural Changes Accompanying Intramolecular Electron Transfer: Focus on Twisted Intramolecular Charge-Transfer States and Structures. Chem. Rev. 2003, 103, 3899-4032.

(44) Kim, H. M.; Cho, B. R. Small-Molecule Two-Photon Probes for Bioimaging Applications. Chem. Rev. 2015, 115, 5014-5055.

(45) Woo, H. Y.; Liu, B.; Kohler, B.; Korystov, D.; Mikhailovsky, A.; Bazan, G. C. Solvent Effects on the TwoPhoton Absorption of Distyrylbenzene Chromophores. J. Am. Chem. Soc. 2005, 127, 14721-14729.

(46) Padilha, L. A.; Webster, S.; Przhonska, O. V.; Hu, H. H.; Peceli, D.; Rosch, J. L.; Bondar, M. V.; Gerasov, A. O.; Kovtun, Y. P.; Shandura, M. P.et al. Nonlinear Absorption in Series of Donor-Pi-Acceptor Cyanines with Different Conjugation Lengths. Journal of Materials Chemistry 2009, 19, 7503-7513.

(47) Luo, S.; Zhang, E.; Su, Y.; Cheng, T.; Shi, C. A Review of Nir Dyes in Cancer Targeting and Imaging. Biomaterials 2011, 32, 7127-7138.

(48) Humeniuk, H. V.; Rosspeintner, A.; Licari, G.; Kilin, V.; Bonacina, L.; Vauthey, E.; Sakai, N.; Matile, S. WhiteFluorescent Dual-Emission Mechanosensitive Membrane Probes that Function by Bending Rather than Twisting. Angew. Chem. Int. Ed. 2018, 57, 10559-10563.

(49) Kreder, R.; Oncul, S.; Kucherak, O. A.; Pyrshev, K. A.; Real, E.; Mely, Y.; Klymchenko, A. S. Blue Fluorogenic Probes for Cell Plasma Membranes Fill the Gap in Multicolour Imaging. RSC Adv. 2015, 5, 22899-22905.

(50) Owen, D. M.; Williamson, D. J.; Magenau, A.; Gaus, K. Sub-Resolution Lipid Domains Exist in the Plasma Membrane and Regulate Protein Diffusion and Distribution. Nat. Commun. 2012, 3, 1256.

(51) Mesmin, B.; Maxfield, F. R. Intracellular Sterol Dynamics. Biochim. Biophys. Acta Mol. Cell Biol. Lipids 2009, 1791, 636-645.

(52) Zidovetzki, R.; Levitan, I. Use of Cyclodextrins to Manipulate Plasma Membrane Cholesterol Content: Evidence, Misconceptions and Control Strategies. Biochim. Biophys. Acta Biomembr. 2007, 1768, 13111324.

(53) Lange, Y.; Ye, J.; Rigney, M.; Steck, T. L. Regulation of Endoplasmic Reticulum Cholesterol by Plasma Membrane Cholesterol. J. Lipid Res. 1999, 40, 2264-2270.

(54) Rambold, Angelika S.; Cohen, S.; Lippincott-Schwartz, J. Fatty Acid Trafficking in Starved Cells: Regulation by Lipid Droplet Lipolysis, Autophagy, and Mitochondrial Fusion Dynamics. Dev. Cell 2015, 32, 678-692.

(55) Filomeni, G.; De Zio, D.; Cecconi, F. Oxidative Stress and Autophagy: the Clash Between Damage and Metabolic Needs. Cell Death Differ. 2015, 22, 377-388.

(56) Cabodevilla, A. G.; Sanchez-Caballero, L.; Nintou, E.; Boiadjieva, V. G.; Picatoste, F.; Gubern, A.; Claro, E. Cell Survival during Complete Nutrient Deprivation Depends on Lipid Droplet-fueled beta-Oxidation of Fatty Acids. J. Biol. Chem. 2013, 288, 27777-27788.

(57) de la Haba, C.; Palacio, J. R.; Martinez, P.; Morros, A. Effect of Oxidative Stress on Plasma Membrane Fluidity of THP-1 Induced Macrophages. Biochim. Biophys. Acta Biomembr. 2013, 1828, 357-364.

(58) Jarc, E.; Kump, A.; Malavasic, P.; Eichmann, T. O.; Zimmermann, R.; Petan, T. Lipid Droplets Induced by Secreted Phospholipase A(2) and Unsaturated Fatty Acids Protect Breast Cancer Cells from Nutrient and Lipotoxic Stress. Biochim. Biophys. Acta Mol. Cell Biol. Lipids 2018, 1863, 247-265.

(59) Bailey, A. P.; Koster, G.; Guillermier, C.; Hirst, E. M. A.; MacRae, J. I.; Lechene, C. P.; Postle, A. D.; Gould, A. P. Antioxidant Role for Lipid Droplets in a Stem Cell Niche of Drosophila. Cell 2015, 163, 340-353. 